\title{
Study on the Network Virtual Ear Experiment Based on 3D Engine
}

\author{
Xiujuan Wang, Dan Wang \& Hui Wang \\ College of Information Engineering, Taishan Medical University, Tai'an 271016, China \\ E-mail: 1975wxj@163.com \\ Lingge Meng \\ Zhangqiu Teachers' College for Further Education, Ji'an 250200, China
}

Received: February 18, 2011

Accepted: March 18, 2011

doi:10.5539/mas.v5n3p221

\begin{abstract}
At present, the virtual experiment has been one of the most important and hot applications in the virtual reality technology. By applying advanced computer and network technology into the classroom teaching and network distance teaching, combining with the ear experiment in the anthropotomy course, using 3DMax and MaxScript scripting language to construct the 3D modeling of ear, and transferring VRML and JavaScript technological combination to realize the dynamic interaction of the ear structure and eardrum vibration. The virtual experiment based on the campus network in the article could be presented to teachers and students by the web form, and support the classroom teaching and network distance teaching sufficiently.
\end{abstract}

Keywords: Virtual experiment, Eardrum, 3D modeling, Dynamic interaction

At present, the virtual experiment has been one of the most important and hot applications in the virtual reality technology. The virtual experiment teaching could break the limitations such as time, place, and equipment quantity, and students could perform the experiment in the more safe and reliable environment, with fewer worries and larger freedom, which could effectively strengthen learners' study interests, handing ability, and abilities of analyzing and solving problems (Shi, 2010). Based on the analysis of relative literatures (Sun, 2006 \& Gong, 2005 \& Nie, 2006 \& Wang, 2009 \& Zhai, 2004 \& Zhou, 2007 \& Wang, 2009), combining with the technical advantages and the practical teaching requirements in the college, the design and practice of the ear experiment in the medical anthropotomy course is studied in this article.

The experiment includes two parts, i.e. the 3D modeling construction and the dynamic interaction realization, and the flow chart of the technical route is seen in Figure 1.

\section{Construction of 3D model}

The surface of the 3D model constructed by 3DMax is joined by many polygons (most of them are triangles). The modeling of ear includes the extruding, bending, stretching, and modifying of the selected figure, and endowing materials.

In the realization, because the structure of ear is complex, involving many peaks and polygons, the computation capacity to render this complex model is large, and to enhance the refresh rate, the model needs to be simplified. The first way is to simplify the peaks and polygons in the model by the peak deleting method, and this method has quick computation speed and occupies fewer memories. The second way is to get rid of the indentation and the basic option, select the option of "normal", and properly adjust the precision digit when deriving the file of "*.wrl" from 3D model.

To further create vivid ear model, the scripting language of 3DMax, the MaxScript language, is used to realize the interior profile of ear. Generate a box, and modify the box, and add the noise wave, and make it a roughness (codes are seen as follows), and the effect figure is seen in Figure 2.

addModifier \$box01

(NoiseModifier fractal:true

seed:9

scale: 100.0

strength:[0, 0, 50] 
)

\section{Dynamic interaction realization of the virtual scene}

Dynamic interaction is the soul of the virtual laboratory. In the experiment of eardrum vibration, the high-effective interaction between user and figure interface includes (1) user touches the sound source to emit sound wave, (2) the sound wave vibrate the eardrum, (3) the sound wave strength increases to break the eardrum. The technical difficulties in the realization will be concretely introduced as follows.

\subsection{User touches the sound source to emit sound wave}

Three parameters in the visual sources of the Sound node in VRML are set up to offer the sound source, i.e. url (set up the linkage address of the sound source), startTime (set up the sounding time of the sound source is -1 , and -1 denotes that the sound source is silent when the interface is opened, and user could control the begin time of the sound source), and loop (set up whether the sound is cycle).

Add a touch sensor to receive the state that user triggers the event for the node of "Sound", and transfer the JaveScript to respond the state that user triggers the event.

\subsection{Sound vibrate the eardrum}

How to make sound vibrate the eardrum? It is hard to realize, but the sound source could control the vibration of eardrum, and the essential is to use the touch sensor to control the sounding and vibrating at the same time, and the sound source will be the switch to control these two actions, and for users, the effects are same. Method 1: Use 3DMax to make the vibration effect of eardrum, and derive the effect to VRML, and set up the start parameter "startTime" is -1 . But this method is hard to control the vibration frequency of eardrum, and the vibration frequency will change with the change of the sound wave frequency. Method 2: In VRML, the sine function " $\sin (\mathrm{x})$ " in the Math function could realize this function. In VRML, there are three parameters in the translation field, i.e. $\mathrm{x}, \mathrm{y}, \mathrm{z}$, and they respectively denote the coordinates of $\mathrm{x}$ axis, $\mathrm{y}$ axis, and $\mathrm{z}$ axis of the object. To realize the changeable vibration frequency of eardrum, the coordinates of the $y$ axis and $\mathrm{z}$ axis should be fixed, and the coordinate of the $\mathrm{x}$ axis could be set up as "Math.sin(x)".

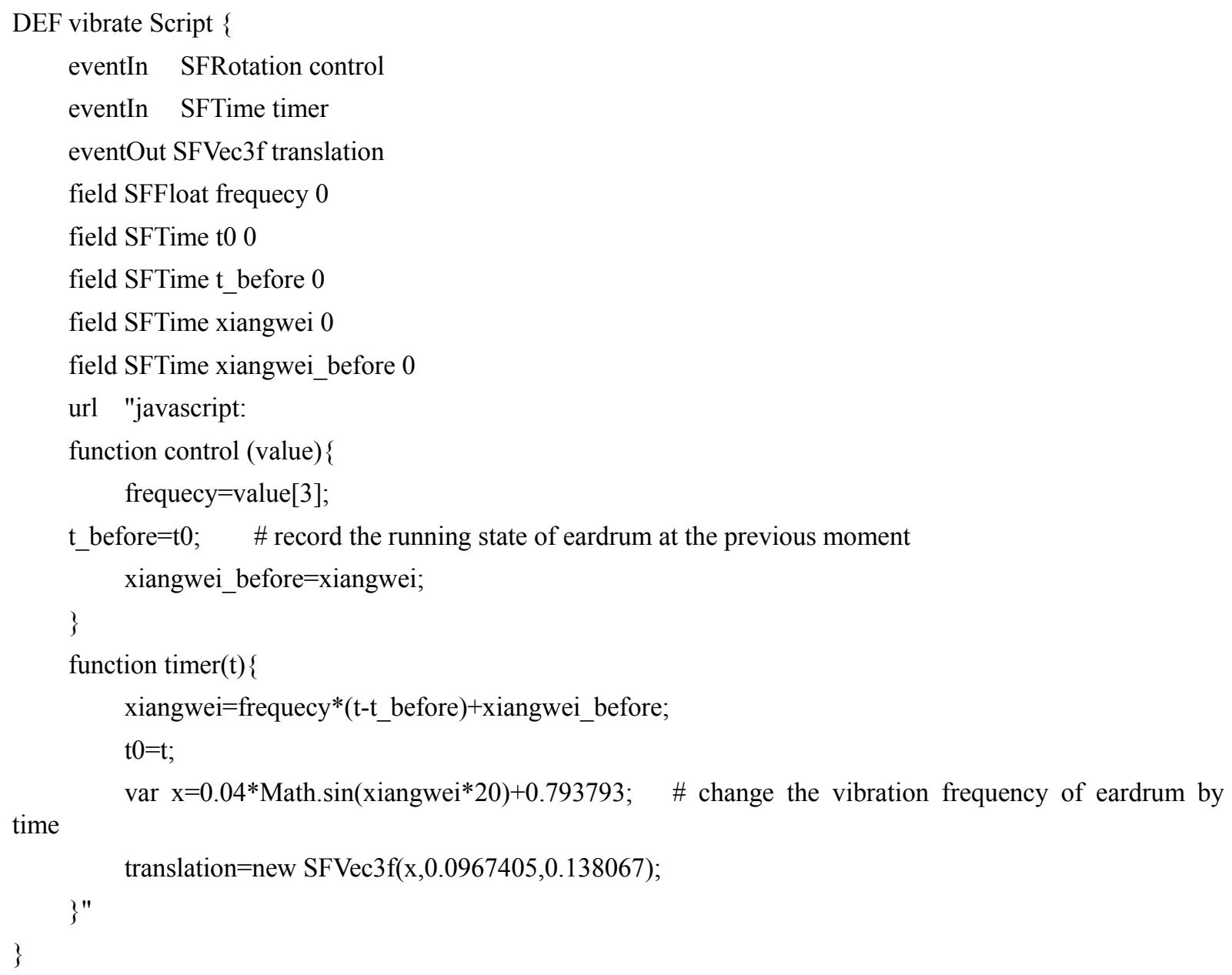




\subsection{Increase the sound wave strength to break the eardrum}

This is the most difficult part in the experiment realization. In VRML, the key to write the codes to generate vivid breaking effect is to transfer the coordinate interpolator and the scalar interpolator, and change the coordinate of the object with the time, combining with the time sensor.

DEF Destory CoordinateInterpolator \{

key $[\ldots .$.

keyValue $[. .$.

\}

DEF Destory ScalarInterpolator \{

key $[\ldots .$.

keyValue $[\ldots$.

\}

But this method is hard to control because of complex writing and difficulty, so the feasible way is to derive the file of "*.wrl" to the VRML after making the eardrum breaking cartoon by 3DMax.

The next step is to judge whether the field of "intesity" of the node of "sound" achieve the maximum 1.0, i.e. if (intensity $==1.0) \quad\{$ start = run; $\}$ ", the action begins to perform, and the eardrum break. If the "loop" is set up as "FALSE", the action is not cycle, and then the program adjusts the size of sound, and the eardrum doesn't vibrate.

\section{Conclusions}

The research in this article is the virtual experiment of pure software based on the campus network. This experiment is realized by depending on many technologies such as 3DMax, VRML, and JavaScript, and presents the result to teachers and students by the web form, and it could effectively support the classroom teaching and the network distance teaching. In the design and realization of the experiment, the experts of anatomy instruct the experiment for the correct direction in knowledge, and the constructivism and the distance teaching interaction theory guarantee the maximum teaching effect of virtual experiment.

\section{References}

Gong, Chenglong, Feng, Yuan \& Wang, Jingzhuo. (2005). The Design and Management of an Online Laboratory for Electronics and Engineering. Distance Education in China. No.3.

Nie, Bin \& Zeng, Zhaofang. (2006). Construction of Virtual Medical Laboratory Based on Virtual Reality Technology. Northwest Medical Education. No.1.

Shi, Lei, Hao, Tinglei \& Lin, Zhuying. (2010). A Study of Interaction Model in Virtual Laboratory Powered by 3D Engine. Distance Education in China. No.8.

Sun, Junfeng, You, Yunhua \& Lu, Jie et al. (2006). Application of VRML in Animal Dissection Virtual Experiment. Computer Engineering and Design. No.11.

Wang, Rongzhi \& Xin, Rihua. (2009). Interaction Design on Interface of Web-based Virtual Experiment. Research and Exploration in Laboratory. No.2.

Wang, Xiujuan. (2009). Study on the Medical Virtual Laboratory Based on the Campus Network. China Science and Technology Information. No.11.

Zhai, Xufeng, Zhu, Yingiie \& Pan, Zhigeng. (2004). 3DX Max Modeling and Its Application in Virtual Reality. Computer Simulation. No.21(4).

Zhou, Bo, Wen, Min \& Li, Hongwei et al. (2007). Experiences of Using the Virtual Reality Technolgoy to Make the Teaching Courseware of Anatomy. Chinese Journal of Anatomy. No.5. 


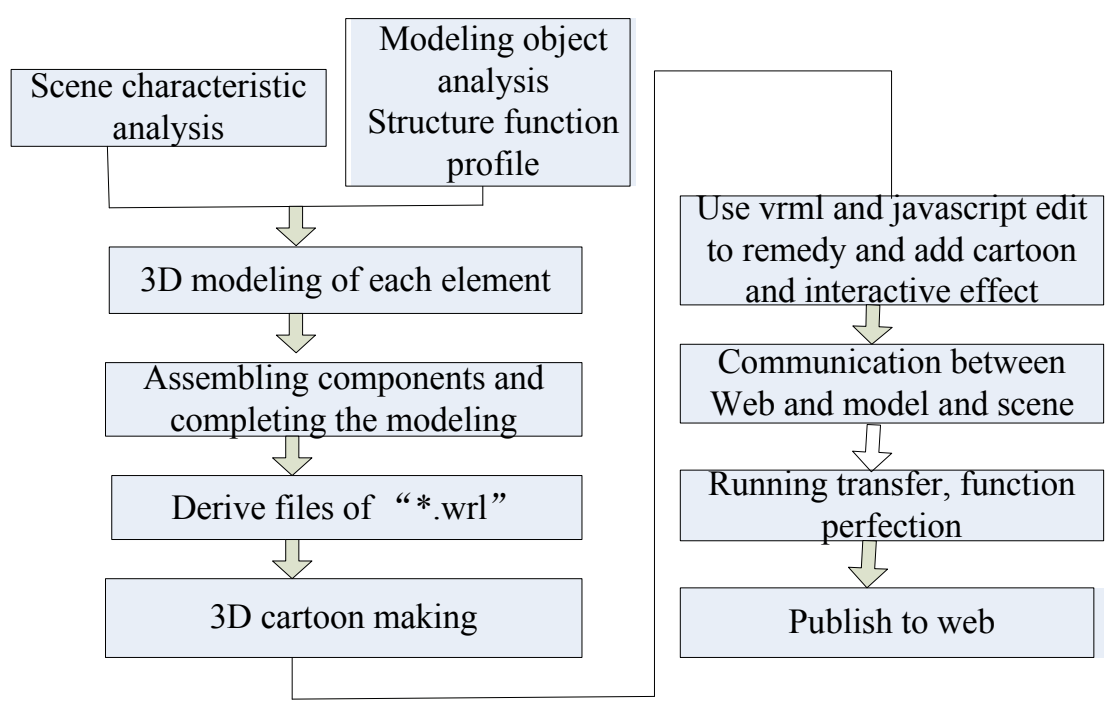

Figure 1. Technical Route Flow Chart of the Virtual Ear Experiment

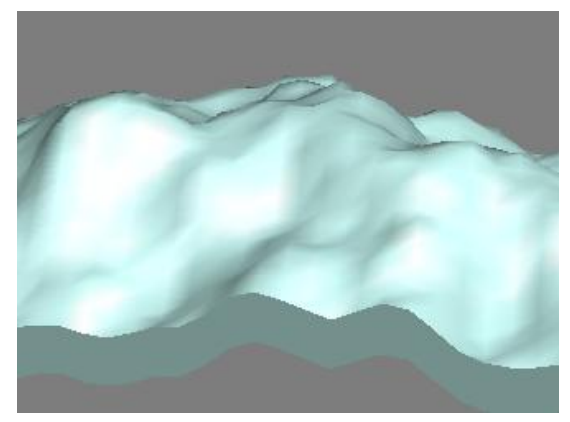

Figure 2. Interior Skeleton Map of Ear 